\title{
Nanomaterials modulate stem cell differentiation: biological interaction and underlying mechanisms
}

Min Wei ${ }^{1,2}$, Song $\mathrm{Li}^{1,2}$ and Weidong $L \mathrm{e}^{1,2,3^{*}}$

\begin{abstract}
Stem cells are unspecialized cells that have the potential for self-renewal and differentiation into more specialized cell types. The chemical and physical properties of surrounding microenvironment contribute to the growth and differentiation of stem cells and consequently play crucial roles in the regulation of stem cells' fate. Nanomaterials hold great promise in biological and biomedical fields owing to their unique properties, such as controllable particle size, facile synthesis, large surface-to-volume ratio, tunable surface chemistry, and biocompatibility. Over the recent years, accumulating evidence has shown that nanomaterials can facilitate stem cell proliferation and differentiation, and great effort is undertaken to explore their possible modulating manners and mechanisms on stem cell differentiation. In present review, we summarize recent progress in the regulating potential of various nanomaterials on stem cell differentiation and discuss the possible cell uptake, biological interaction and underlying mechanisms.
\end{abstract}

Keywords: Nanomaterials, Stem cells, Differentiation

\section{Background}

Stem cells are primitive cells that have the potential to self-renew and develop into different specialized functional cells. According to its developmental stage, stem cells can be classified into two broad types, embryonic stem cells (ESCs) and somatic stem cells (SSCs) [1]. ESCs are derived from the inner cell mass of blastocysts [2, 3]. With similar characteristics of ESCs, induced pluripotent stem cells (iPSCs) are produced from somatic cells by genetically reprogrammed to a ESCs-like state by introducing the expression of certain genes and factors [4]. ESCs and iPSCs are pluripotent stem cells that have the greatest differentiation potential and infinite self-renewal capacity [5, 6]. SSCs, derived from adult tissues, are more accessible, but less potent than ESCs and iPSCs [7]. In recent years, with the continuous research of stem cells, more and more types of SSCs can be isolated from bone marrow, adipose tissues, cord blood and neural tissues

\footnotetext{
*Correspondence: wdle@sibs.ac.cn

${ }^{1}$ Liaoning Provincial Center for Clinical Research on Neurological Diseases, The First Affiliated Hospital, Dalian Medical University, Dalian 116021, People's Republic of China

Full list of author information is available at the end of the article
}

[8-11]. Mesenchymal stem cells (MSCs) and adiposederived stem cells (ADSCs) as well as neural stem cells (NSCs) have become attractive stem cell source for tissue regeneration and engineering without considering the ethical issues of ESCs [12].

The clinical application of stem cells, especially in cell therapy and tissue engineering, depends on the regulation and control of cell differentiation into specific cell types [13]. In the past decade, great efforts have been made to manipulate the differentiation of stem cells into numerous types of cells, such as osteoblast cells, neurocytes, adipocytes and cardiomyocytes [14-16]. However, the low differentiation efficiency and success rate limits the development of stem cell differentiation for stem cell therapy. Additionally, undifferentiated ESCs after implantation in vivo increase the risk of teratoma, so it is important to allow committed differentiation of ESCs into specific lineages prior implantation for a safe use in cellbased therapies $[17,18]$. Thus, there is an urgent need to develop strategies to improve the efficiency of directed differentiation of stem cells into specified cell types.

Nanomaterials are materials with a microstructure the characteristic length scale (at least one dimension) 
of which is within the nanometer range $(\sim 1-100 \mathrm{~nm})$. Nanomaterials have been widely used to manipulate the cell behavior due to their small size, ease of synthesis and versatility in surface functionalization [19-21]. During the last decade, various nanomaterials, including liposomes [22], quantum dots [23, 24], carbon nanotubes (CNTs) [25], graphene (GR) [26], silica nanoparticles [27], titanium dioxide nanoparticles $\left(\mathrm{TiO}_{2}\right)$ [28], silver nanoparticles (AgNPs) [29], gold nanoparticles (AuNPs) [30], iron oxide nanoparticles (IONPs) [31], DNA nanostructures [32], have been intensively explored in both biological and medical fields.

The rapid development of nanotechnology provides a great prospect for the development of novel nanomaterials with modulating potential on stem cell differentiation. In fact, various types of nanomaterials have been identified to regulate the differentiation of stem cells (i.e. ESCs, iPSCs and MSCs) into different types of cells, including adipocytes, cardiomyocytes, osteoblast cells, and neural cells through different mechanisms [33-37]. The extracellular microenvironment is considered to play an important role in influencing the function and fate of stem cells [11]. Engineered nanomaterials can mimic the stem cell environment and modulate stem cell differentiation [38]. The suppletion of specific differentiation factors such as growth factors and bioactive molecules into the medium is the widely accepted route to promote stem cell differentiation [39]. Recently, accumulating evidence has indicated that some nanomaterials, such as functionalized CNTs and GR, can facilitate stem cell proliferation and differentiation even without the need of specific media containing extra supplements [40, 41]. Furthermore, nanomaterials with surface chemical modifications can also modulate the specific properties of stem cells for differentiation. In this review, we summarize recent research progress in the modulating effects of nanomaterials on stem cell differentiation and discuss the possible modulating manners and underlying mechanisms.

\section{Nanomaterials-modulated stem cell differentiation Metal nanoparticles}

AuNPs Due to their intrinsic properties such as wellcontrolled size and surface- functionalization, AuNPs have been widely used in biomedical fields for drug/gene delivery, biosensors, imaging, and photothermal therapy $[42,43]$. The internalized AuNPs (with different surface modification or payload) may interact with proteins located in the cytoplasm, or serve as mechanical stimuli that trigger a series of biological alterations and modulate cell behaviors [34, 44, 45]. The cellular effects of AuNPs on the differentiation of stem cells have been investigated; various forms of AuNPs (sizes in 20-70 nm, surface modified with citrate, chitosan or fibronectin, etc.) have been reported to modulate the differentiation of stem cells (ADSCs, MSCs, ESCs, MSCs) into osteoblasts or cardiomyocytes [34, 46-49].

Increasing evidence suggests that AuNPs possess an inherent ability to promote the differentiation of stem cells. The size, shape and surface modifications of AuNPs can impact the cellular uptake of particles into stem cells, and consequently influence their modulating effects on stem cell differentiation. For instance, 30 and $50 \mathrm{~nm}$ sphere AuNPs have been proved to be most efficient among all sizes on osteogenic differentiation of hADSCs, while 40 and $70 \mathrm{~nm}$ sphere AuNPs, $70 \mathrm{~nm}$ Au nanorods coated with bovine serum albumin (BSA) affect the osteogenic differentiation of hMSCs obviously [44, 47]. In addition to the intrinsic properties of AuNPs themselves, charge and specific chemical moieties on nanomaterials surface may also contribute remarkably towards direction of stem cell fate [50]. Nanomaterials with modified surfaces can also be chemically altered to improve specific properties for enhanced cell-matrix interactions. For example, chitosan-conjugated AuNPs can promote the osteogenic differentiation of human ADSCs (hAD$\mathrm{SCs})$ through the $\mathrm{Wnt} / \beta$-catenin signaling pathway [49]. Fibronectin-coated AuNPs as adhesion sites deliver electrical stimulation on the differentiation of human ESCs (hESCs) in vitro and direct induce osteogenic differentiation [48]. Additionally, AuNPs can also be utilized for cardiac differentiation. AuNPs-loaded functionalized nanofibers scaffold can couple adequate electrical, mechanical, biological or chemical properties, leading to enhanced cardiomyogenic differentiation of hMSCs [46, 51]. As for the underlying molecular mechanisms, further study has shown that AuNPs promote osteogenic differentiation of mouse MSCs (mMSCs) through p38 mitogen-activated protein kinase (MAPK) pathway [34]. Notably, although AuNPs with different sizes have been tried for stem cell differentiation, their side effects should not be neglected due to their non-biodegradable nature [52]. AuNPs modified onto three-dimension (3D) scaffolds to deliver electrical stimulation for specific stem cell differentiation seems to be a more reliable method.

AgNPs Due to its remarkable antibacterial activity, AgNPs have been widely used and have become one of the fastest growing nanomaterials in the biomedical fields of recent years [53]. Similar to AuNPs, AgNPs also face the challenge that they are non-biodegradable materials and cannot be degraded in cells. The role of AgNPs in the differentiation of stem cells is controversial. AgNPs (10 or $20 \mathrm{~nm}$ in size) show no influence on the differentiation of hADSCs and caused minimal toxicity at antimicrobial concentrations [54]. In contrast, $30 \mathrm{~nm}$ AgNPs do not influence the osteogenic differentiation of hMSCs, 
but cause certain cytotoxicity [55]. Furthermore, AgNPs has been reported to inhibit stem cell differentiation, both AgNPs with a size of $80 \mathrm{~nm}$ (hydro-dynamic diameter) and silver ions could attenuate the differentiation of hMSCs to adipogenic and osteogenic even at non-toxic concentrations [56]. In contrast, another experiment leads to the opposite conclusion that AgNPs can promote osteogenic differentiation of human urine-derived stem cells (hUSCs) at a suitable concentration, in a silver ionsindependent manner [57]. In addition, it can also promote the proliferation and osteogenic differentiation of mMSCs in vitro [29]. These conflicting conclusions require us to be cautious about the application of AgNPs in stem cell differentiation. Further studies are needed to find the exact underlying mechanisms. Meanwhile, AgNPs can be fabricated as drug delivery vehicles to deliver light-activated miR-148b mimic, and these miR-148-AgNPs conjugates are readily entering into cells and resulting in differentiation of hADSCs into an osteogenic linage [58]. All these findings confirm that different sizes and concentrations of AgNPs may have different effects on stem cell differentiation; therefore suitable size and concentration as well as surface modification are critical manners in consideration of application of AgNPs in stem cell differentiation.

$\mathrm{TiO}_{2}$ In view of the good biocompatibility and highly ordered nanotube arrays structure, the ability of $\mathrm{TiO}_{2}$ nanoparticles to promote the stem cell differentiation have attracted much attention $[35,36]$. Several studies have shown that $\mathrm{TiO}_{2}$ of specific size and shape may have a certain effect on stem cell differentiation. The spherical $\mathrm{TiO}_{2}$ nanoparticles have been reported to exert negative effects on cell viability and negatively affect the osteogenic differentiation of rat MSCs (rMSCs) in a size- and dosedependent manner [59]. In contrast with the inhibiting effects of $\mathrm{TiO}_{2}$ nanoparticles on stem cell differentiation, $\mathrm{TiO}_{2}$ nanotubes have been documented to promote stem cell differentiation [36]. Since the diameter of $\mathrm{TiO}_{2}$ nanotubes can be synthesized variably, nanoscale geometry has been shown to influence cellular differentiation. Studies have been conducted to determine the optimal sizes of $\mathrm{TiO}_{2}$ nanomaterials for their regulation on cell differentiation [60]. For example, $15 \mathrm{~nm}$ has been indicated as an optimal length for $\mathrm{TiO}_{2}$ nanotube to modulate adhesion and differentiation of human hematopoietic stem cells (hHSCs) [61]. However, another one study conducted by Lv et al. [35] has demonstrated that compared with 50 and $100 \mathrm{~nm}$ in size, $70 \mathrm{~nm}$ is the optimal dimension for $\mathrm{TiO}_{2}$ to regulate osteogenic differentiation both in vitro and in vivo. The $\mathrm{TiO}_{2}$ induces differentiation of hADSCs via an epigenetic mechanism by modulating histone $\mathrm{H} 3$ at lysine 4 trimethylation. Further study on underlying molecular mechanisms between $\mathrm{TiO}_{2}$ nanotubes and stem cell differentiation has revealed that $\mathrm{Na}^{+} / \mathrm{K}^{+}$transporting ATPases ATP1A2 (alpha 2 polypeptide), ATP1A3 (alpha 3 polypeptide) and mitogen-activated protein kinase kinase kinase 11 (MAP3K11) are involved in the $100 \mathrm{~nm} \mathrm{TiO}_{2}$ nanotubes-induced osteogenic differentiation of rat bone marrow stromal cells [62]. Besides, $\mathrm{TiO}_{2}$ nanotube arrays covered with gelatin/chitosan multilayered coatings can be used as drug nanoreservoirs for bone morphogenetic protein 2 (BMP2) loading. The multilayer-coated $\mathrm{TiO}_{2}$ nanotube arrays are able to promote the osteoblastic differentiation of rMSCs for controlled BMP2 release [63]. In addition, $\mathrm{TiO}_{2}$ can fabricate as $3 \mathrm{D}$ scaffolds for stem cell differentiation. $\mathrm{TiO}_{2} 3 \mathrm{D}$ scaffolds coated with alginate hydrogel containing simvastatin or enamel matrix derivative can direct osteogenic differentiation of stem cells [14, 64]. These findings above suggest that the shape and size of $\mathrm{TiO}_{2}$ nanoparticles has a great influence on the differentiation of stem cells, and $\mathrm{TiO}_{2}$ nanotubes but not spherical $\mathrm{TiO}_{2}$ nanoparticles could serve as good biomaterials for the differentiation of stem cells. In addition, $\mathrm{TiO}_{2}$ is more suitable for use as two-dimensional (2D) substrates or 3D scaffolds for stem cell differentiation.

IONPs Similar to other metal-based nanomaterials, IONPs are also proved suitable for promoting stem cell differentiation. IONPs have been confirmed to promote osteogenic differentiation of human bone-derived mesenchymal stem cells (hBMSCs) in vitro by activating MAPK signal pathway [65]. Meanwhile, another study has reported that the field-induced assemblies of magnetic $\gamma-\mathrm{Fe}_{2} \mathrm{O}_{3}$ nanoparticles can promote the differentiation of primary mouse bone marrow cells into osteoblasts [66]. The advantage of this approach is that the promoted differentiation effect is mediated by interface effect rather than internalization. Furthermore, IONPs coated with human serum albumin (HSA) can be used as non-toxic and superparamagnetic delivery for drug controlled release. Conjugated fibroblast growth factor 2 (FGF2) core-shell NIR fluorescent iron oxide/HSA magnetic nanoparticles are also effective in enhancing the proliferation of $\mathrm{hBM}$ SCs and promoting their differentiation toward neuronal, adipogenic or osteogenic lineages in vitro [67].

Other metal-based nanomaterials Barium titanate nanoparticles with glycol-chitosan coating have a good biocompatibility to promote the ostogenic differentiation of rMSCs in the presence of the appropriate differentiation factors (osteogenic differentiation basal medium supplemented with dexamethasone, GA-1000 (gentamicin, amphotericin-B), L-glutamine, ascorbate, FBS and glycerophosphate) [68]. Additionally, metal-based composite nanomaterials have been tested for stem cell differentiation. Magnetic core-shell structures with a $\mathrm{ZnFe}_{2} \mathrm{O}_{4}$ core 
surrounded by a gold outer shell have been successfully utilized to deliver specific siRNA/pDNA to selectively direct the differentiation of NSCs into neurons or oligodendrocytes [69].

\section{Carbon nanomaterials}

Carbon nanomaterials, including CNTs, GR and carbon 60 (C60), are novel materials which have been widely studied and applied [70]. These new carbon materials possess several excellent physical and chemical properties, and have been applications in biological sensors, gene and drug delivery and stem cell tracking [71, 72]. In order to meet the needs of the strong demand for the discovery and development of stem cell differentiation modulator, many carbon nanomaterials have been evaluated and showed significant modulating effects on stem cell differentiation.

GR and graphene oxide (GO) GR is a novel 2D carbon nanosheet with unique physical, chemical and mechanical properties that are widely used in biomedicine field [72]. GR and its derivative, GO, have recently attracted increasing interests for biology applications. GO is an oxidative derivative of GR. Epoxide, hydroxyl, carbonyl and carboxyl groups are on the basal planes and edges of GO sheet, providing strong bonding sites and bioactivities [73, 74]. GR and GO are demonstrated to be the ideal biocompatible and mechanical platforms mediating stem cells growth and differentiation [16]. Remarkably, the different surface properties of GO and GR exhibit distinct characteristics for modulating mouse iPSCs proliferation and spontaneous differentiation. GO accelerates the iPSCs differentiation, whereas GR favorably maintains the cells in an undifferentiated state [75].

GO and GR have been reported to promote the differentiation of stem cells to neurons. GO can effectively promote the differentiation of $\mathrm{mESCs}$ to dopamine neuron after induction of stromal cell-derived inducing activity (SDIA) [33], while GR can be used as a cell-adhesion layer for long-term differentiation of hNSCs toward neurons [76]. Meanwhile, 3D rGO-collagen hybrid scaffold is good for the enhancement of the neural differentiation of rBMSCs [77].

Moreover, owing to their ultra lightweight, tremendous strength and stability, GR and GO also have been emerged as promising nanomaterials for tissue engineering. GO and GR sheets have the potential to support and accelerate stem cell adhesion, proliferation and differentiation, such as facilitate hMSCs differentiation towards osteogenic lineage [16]. Consistently, GR provides a promising biocompatible scaffold that does not hamper the proliferation of hMSCs and accelerates their specific differentiation into bone cells even in the absence of commonly used additional growth factors such as BMP-2 [41]. Furthermore, GO-doped poly(lactic-coglycolic acid) (PLGA) nanofiber designed as 3D scaffolds effectively promote the differentiation of hMSCs toward osteoblasts. The incorporated $\mathrm{GO}$ can enhance the hydrophilicity and protein-/inducer adsorption ability of the nanofibers. It not only accelerates the attachment and proliferation of hMSCs, but also induces the osteogenic differentiation [78].

Additionally, as a new type of carbon-based quantum dots, graphene quantum dots (GQD) also exert no significant influences on self-renewal potential and enhance the differentiation of rBMSCs into osteoblasts and adipocytes [79].

CNTs CNTs have emerged as one of the most promising nanomaterials due to their tremendous strength, ultralight weight and high stability. CNTs can be considered as a layer of rolled GR sheet [80]. According to the numbers of GR layers, they are normally categorized as singlewalled (SWNTs) or multi-walled nanotubes (MWNTs) [81]. Both SWNTs and MWNTs have no adverse effects on biocompatibility, proliferation, or differentiation of hMSC for future approaches to tissue repair/regeneration [82]. However, carboxylated-CNTs have been demonstrated to inhibit the proliferation, osteogenic/adipogenic differentiation of mMSCs in a suspended CNTs condition [83]. These conflicting results may indicate a negative impact of surface modification on CNTs-modulated differentiation of stem cells.

CNTs are good matrix materials for stem cell differentiation. Polyethylene glycol (PEG)-conjugated MWNTs layers show no cytotoxicity $[40,84,85]$, and accelerate the differentiation of stem cells even without any additional differentiating factors [40]. In another study, carboxylated MWCNT-coated substrates have been reported to provide a suitable environment for the spontaneous long-term neural differentiation of hBMSCs with low cytotoxicity [86]. Also, SWCNTs films are excellent 2D nanomaterials that can enhance the cell growth and differentiation of rMSCs as a culture substrate; the variation of thickness, roughness, surface property of SWCNTs films will positively affect the growth and differentiation characteristics of MSCs, and specific cells differentiated from MSCs can be precisely controlled by altering the property of SWCNTs films [87]. Incorporated carbon nanomaterials (a mixture of GR and SWCNTs) into electrospun polycaprolactone (PCL) scaffolds can greatly improve the mechanical strength properties of the scaffolds and enhance hBMSCs growth and chondrogenic differentiation [88].

CNTs can also be designed and fabricated as novel 3D nanostructured scaffolds for stem cell proliferation 
and differentiation. Hydrogen treated CNTs poly(L-lactic acid) scaffolds with poly-L-lysine (PLL) coating can induce the differentiation of hBMSCs into chondrogenic more than control groups [89]. Furthermore, poly( $\varepsilon$ caprolactone)-CNTs composite scaffolds possess the ability to promote cardiac differentiation of hMSCs in 3D culture [90]. The incorporated CNTs can greatly improve the strength of composite scaffolds, and possess an inherent ability to promote stem cell differentiation without adverse effects on cellular activity. Despite the advantage for stem cell differentiation mentioned above, CNTs have some limitations. Pristine CNTs are insoluble and cannot be used directly; moreover, nanotubes also have some toxic effects [91]. Proper surface modification of CNTs can increase solubility and reduce toxicity, which should be taken into account when CNTs are used for stem cell differentiation.

\section{Semiconductor nanomaterials}

As an important semiconductor nanomaterial, silica nanoparticles can serve as a vehicle for drug delivery or gene therapy [27, 92]. It seems that silica nanoparticles lack of positive effect but even have negative effects to some extent on stem cell differentiation [93]. Previous studies have shown that the pure nanoparticles had no effect on cellular ultrastructures and adipogenic/osteogenic differentiation of rMSCs [94]. However, due to its biocompatibility, controllable particle size, tunable pore size and high loading capacity, silica nanoparticles have been explored to serve as nanocarriers to promote the stem cell differentiation [92]. Silica nanoparticles are good protein carriers and can be used as carriers for insulin delivery to induce the rMSCs differentiate into adipocytes in vitro [94]. Furthermore, the treatment of hESCs with ascorbic acid (AA)-loaded fluorescent TRITCmesoporous silica nanoparticles results in a higher induction efficiency of stem cell differentiation and can serve as a potential tool to promote the differentiation of hESCs into cardiomyocytes [15]. Additionally, silica nanoparticles as nucleic acid carriers are also used for specific differentiation. FITC-conjugated mesoporous silica nanoparticles are fabricated as a suitable carrier to deliver hepatocyte nuclear factor $3 \beta$ (HNF3 $\beta$ ) plasmid DNA, the silica-based delivery platform can quickly induce mouse iPSCs to differentiate into hepatocyte-like cells [95]. In addition, silica also can be modified as nanocarriers to deliver pigment epithelium-derived factor (PEDF) siRNA to regulate the differentiation and self-renewal of cardiac stem cells [96]. Although silica nanoparticles itself does not have the ability to promote stem cell differentiation, it is worth mentioning that silica nanoparticles can be considered as a nanocarrier for stem cell differentiation applications due to their good bio-safety, biocompatibility, and high loading capacity.

\section{Polymeric nanoparticles}

Polymeric nanoparticles also play an important role in the differentiation of stem cells. Polymeric nanoparticles are capable of modifying active drugs, delaying and controlling the release of drugs, and being frequently used as drug-delivery systems [97]. Retinoic Acid (RA) can be controlled release from RA-hydrophilic polyethylenimine (PEI) complex nanoparticles in vitro by $\mathrm{pH}$ and induce the ES cell-derived neuronal differentiation [98]. Moreover, thermo-responsive RA-loaded poly ( $N$-isopropylacrylamide)-co-acrylamide (PNIPAM-co$\mathrm{Am})$ polymeric nanoparticles (PCANs) can enhance hiPSC differentiation. RA can be intracellular released from RA-loaded PCANs for the thermo-responsive property and efficiently direct hiPSC differentiation into neuronal lineage [99]. Particularly, polyelectrolyte nanoparticles by electrostatic interaction of PEI and dextran sulfate can deliver RA into mouse sub-ventricular zone (SVZ) stem cells in vitro, the results have demonstrated that the RA-loaded nanoparticles can intracellular release RA and promote the differentiation of stem/ progenitor cells [100]. Furthermore, their further study has shown that the RA-loaded polymeric nanoparticles could be in vivo control the differentiation of SVZ neural stem cells, the differentiation mechanism of RA-loaded nanoparticles is that the released RA from nanoparticles interacts with RA receptor (RAR), activate SAPK/JNK signaling pathway, and ultimately increase the expression of proneurogenic gene [101]. These findings may pave the way for the treatment of neurodegenerative diseases by using nanomaterials and make polymeric nanoparticles a useful delivery system of RA for neuronal differentiation.

Among the polymeric nanoparticles, chitosan offers certain advantages over other ones for drug delivery due to its biodegradability, biocompatibility, low immunogenicity and low toxicity [102]. Delivery of nucleic acid with chitosan to regulate osteogenic differentiation was also tested. Chitosan nanoparticle/hsa-miR-199a-5p agomir complexes can both modulate osteogenic differentiation of hMSCs in vitro and improve the regeneration of bone in vivo in a hypoxia inducible factor- $1 \alpha$ (HIF-1 $\alpha$ ) pathway related manner [103]. It has been documented in another study that chitosan is a potential candidate as an efficient non-viral miRNA vector to regulate the osteogenic differentiation of MSCs; chitosan-based- microRNA nanoparticles can be a safe and effective carrier for antimiR-138 delivery to rMSCs with high transfection efficiency and significantly enhance the osteogenesis of rMSCs [104]. Direct delivery of miRNA into stem cells 
provides appropriate therapy of specific diseases, the high loading capacity and controlled drug-release ability make polymer nanoparticles become promising drug carriers for various differentiation purpose.

Furthermore, polymers are candidates of highly biocompatible scaffolds for stem cell differentiation. Novel spherical nano-hydroxyapatite/chitosan/gelatin 3D porous scaffolds can also enhance the proliferation and osteogenic differentiation of hiPSCs [105].

Although polymeric nanoparticles have the above advantages in stem cell differentiation, the cytotoxicity of most polymers themselves is still a question that cannot be ignored. Polymers with high molecular weight, such as high branched PEI exhibit high toxicity; low molecular weight display low toxicity yet transfection efficiency is low as well $[106,107]$. Other polymeric nanoparticles like PLL, poly(diallyl-dimethyl-ammonium chloride) show similar toxicity to PEI [108]. However, chitosan and its derivatives display better biocompatibility and relatively good transfection efficiency $[109,110]$. No one system meets all needs; among these polymeric nanoparticles, chitosan seems to have a better biocompatibility, which is valuable to study more.

\section{DNA nanostructures}

DNA nanostructures with well defined structures and uniform sizes have emerged as novel nanomaterials for biomedical applications [32]. Variety of artificial DNA nanostructures, including DNA origami, DNA tetrahedron, DNA nanotube, have been fabricated through appropriate design of DNA sequences [111-113]. DNA nanostructures show merits in low immunogenicity, good biocompatibility, controllable surface modification, reproducibility and low cost. Numerous studies have reported the potential application of DNA nanostructures for disease diagnosis and treatment, especially in the areas of biosensing and drug delivery [32, 111]. DNA nanostructures as artificial scaffolds to control the cell behavior are also tested. Assembled DNA nanotubes by self-assembly and covalently functionalized with the bioactive cell adhesion peptide Arg-Gly-Asp-Ser (RGDS) can used as artificial substrate for guiding the differentiation of mNSCs [114]. Remarkably, both nanotube architecture and peptide bioactivity synergistically promotes mNSCs differentiate into neurons rather than astrocytes [114].

\section{The interactions between nanomaterials and stem cells and possible underlying mechanisms}

Up to now, the effect of nanomaterials on stem cell differentiation has been studied to a large extent by introducing stem cells into artificial microenvironment, and the application of nanomaterials in stem cell differentiation is mainly through the following ways: cell culturing with nanoparticles suspension, 2D cell culture on the surface of nanomaterials, or cell seeding and culturing on 3D nano-scaffolds (Fig. 1).

\section{Nanoparticles as supplements for stem cell differentiation}

Some nanoparticles possess an inherent ability to facilitate stem cell differentiation due to their unique biological and mechanical properties. Up to now, several promising nanoparticles including AuNPs, AgNPs, GO, CNTs and silica nanoparticles have been demonstrated to promote stem cell differentiation [29, 33, 34, 86, 115]. Nanoparticles can easily transfer across cells membranes and locate in the cytoplasm, thus affecting certain cellular signaling pathways for inducing differentiation [116, 117]. The cellular pathways may differ depending on the type of nanomaterials, surface ligands and cell types. The physicochemical features of nanomaterials have a great influence on the mechanism of differentiation (Fig. 2).

Size and shape Nanoparticles can serve as mechanical stimuli to activate certain signaling pathways in stem cells and thus induce differentiation. The optimal nanoparticles size for stem cell differentiation ranges between 20 and $70 \mathrm{~nm}$, which probably due to the size-dependent cellular uptake rates [35, 44, 47]. Nanoparticles around $50 \mathrm{~nm}$ in size showed higher amounts internalized by cells [118]; smaller nanoparticles are more cytotoxic, while larger nanoparticles are less efficiently incorporated by cells $[119,120]$. Furthermore, the shape of nanoparticles affects the uptake of nanoparticles by cells that may influence the stem cell differentiation [44]. The uptake rate of

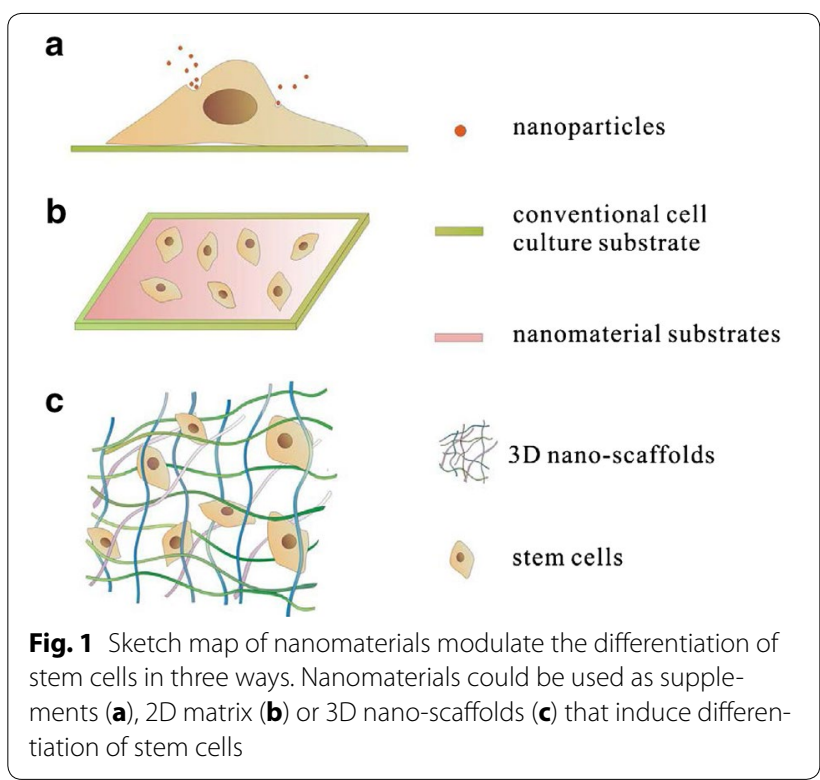




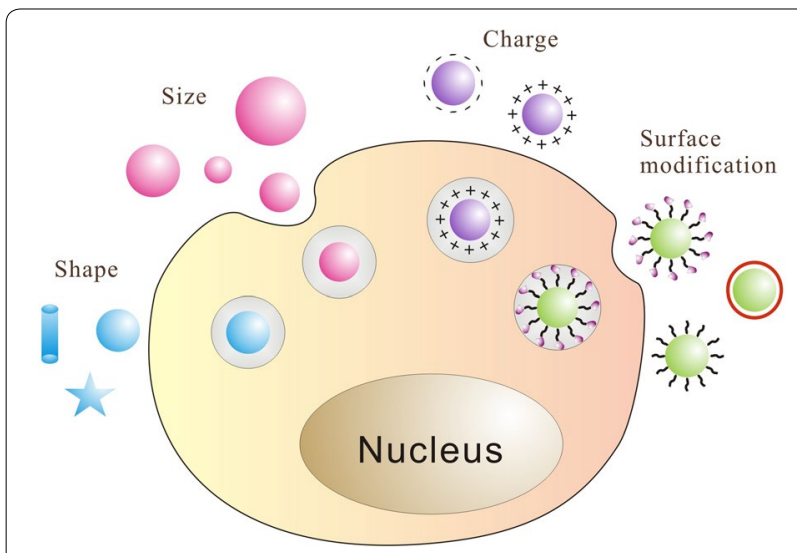

Fig. 2 The physicochemical features of nanomaterials influence cellular uptake and consequently impact their modulating potential on stem cells differentiation

nanospheres is much higher than that of nanorods when their size is approximate [120]. All in all, nanoparticles were taken up by cells in a size and shape-dependent manner, thus further affecting differentiation; nanoparticles would cause mechanical signals on cells and affect stem cell differentiation due to the varying size and shape.

Charge and moiety Charge and specific chemical moieties are also important for the nanomaterials to direct stem cells differentiation. Functional chemical moieties, such as amines $\left(-\mathrm{NH}_{2}\right)$, hydroxyl $(-\mathrm{OH})$ and carboxyl ($\mathrm{COOH})$ are widely present in biomolecules such as proteins, nucleic acids, lipids and polysaccharides, are important factors that affect the behavior and differentiation of stem cells [121]. For example, $\mathrm{COOH}-\mathrm{AuNPs}$ treatment inhibits osteogenic differentiation, whereas those $-\mathrm{NH}_{2}$ and $-\mathrm{OH}$ groups functionalized AuNPs fail to do so [50]. In addition, different surface charges and groups affect the uptake of nanoparticles, and positively charged nanoparticles exhibit higher cell uptake and higher cytotoxicity [120]. It is noteworthy that most physical and chemical parameters are interconnected, the influence of charge may be related to size-dependent uptake, and additional surface coatings add complexity.

Surface modification Different surface coating of nanomaterials can lead to different cell signaling cascades. For example, AuNPs promote osteogenic differentiation of MSCs through the p38 MAPK pathway, while chitosanconjugated AuNPs activate the Wnt/ $\beta$-catenin signaling pathway in hADSCs $[34,49]$. Nanomaterials with specific surface modifications can more closely mimic the microenvironment and interaction with biological molecules and stem cells [50]. Furthermore, nanomaterials can absorb serum proteins or even bioactive molecules such as cytokines, growth factors in the physiological environment, which can promote stem cell differentiation $[4,122$, 123]. In addition, surface charge and the size of nanomaterials will affect the adsorption of differentiation factors, which is due to the influence of different electrostatic interaction and area-to-volume ratio [124].

The interactions of nanoparticles and stem cells have not clearly explained, as most physicochemical parameters are entangled. It remains to further investigate the underlying mechanism of the differences relatively to the internalized nanoparticles for the differentiation of stem cells. It is worth noting that, although these nanomaterials appear to be non-toxic to cells and can promote stem cell differentiation into specific lineages, the long-term biological safety still requires further evaluation due to most of the nanomaterials cannot be degraded after cellular uptake into cells.

\section{As nanocarriers for drug delivery for stem cell differentiation}

Nanomaterials have shown great potential as intracellular nanocarriers for drug and nucleic acid delivery in the differentiation of stem cells. Some of the drug/chemicals have poor solubility, short half-life and/or poor penetration into cells, furthermore, naked nucleic acids cannot successfully enter cells which require the assistance of a suitable vector $[125,126]$. Once inside in the cells, exogenous nucleic acids or biomacromolecules can be quickly degraded by intracellular enzymes, and small-molecule drugs rapidly metabolized by cells [125]. Nanoparticles are ideal carriers for nucleic acids/drug delivery both in vitro and in vivo [127]. Nanoparticles have the advantages of good biocompatible and ease of functionalization that they can target stem cells and release their payloads in the cytoplasm [127]. This unique feature enables nanoparticles to be used as excellent carriers to deliver drugs, nucleic acids, growth factors and other biomolecules within cells for stem cell differentiation [100, 128, 129]. Chitosan is biodegradable nanocarrier to deliver miRNA to regulate the osteogenic differentiation of MSCs, and various polymeric nanoparticles are used to deliver RA [103, 130]. In addition, inorganic nanoparticles such as AuNPs, AgNPs and silica nanoparticles are often used for drug delivery because of their load capacity, although their application is limited due to their non biodegradability [52]. For example, AgNPs is designed as a carrier to deliver miR-148b and mesoporous silica nanoparticles is used for delivery of AA $[15,58]$. Nanoparticles can serve as a platform to carry different bioactive payloads with almost no influence on cell activity but a great impact on differentiation. Thus it can be seen that when drug-loaded nanomaterials enter into cells and release their payloads within cytoplasm, the payloads subsequently activate the corresponding signaling cascade. 
The mechanism of differentiation is mainly determined by their surface payloads of nanomaterials. Remarkably, biocompatible and biodegradable nanoparticles with the ability to target stem cells and release their payloads in the cytoplasm, and then activate signaling cascades, may be the focus of future research [117].

\section{Nanomaterials as 2D matrix support for stem cells growth and differentiation}

2D cell culture is a traditional method of cell culture in vitro. Physical and biological factors, such as growth factors, hormones, chemical or biological molecules, and extracellular matrix, can determine the fate of stem cells of differentiation and pluripotency. Therefore, when cultured on different cell culture substrates, stem cells may have different differentiation fates. Several researchers have reported that using nanomaterials as $2 \mathrm{D}$ cell culture substrate could effectively promote the differentiation of stem cells into specific lineages, and the stiffness, surface chemistry, alignment and several other parameters of the cell culture substrate (matrix) may work together to influence the fate of stem cells $[16,35,61,131]$. Chemical and biological modifications of nanomaterials can directly influence cell-matrix interactions and ultimately manipulate the signal transduction pathways in stem cells. As an example, the aligned CNTs exhibit an enhanced proliferation and osteogenic differentiation of hMSCs probably due to the ordered nanomaterials may better mimic the orderly pattern of natural ECM [131]. Overall, nanomaterials as $2 \mathrm{D}$ matrix with certain geometric properties have shown positive effects on the differentiation of stem cells, which make nanomaterials good candidates for stem cell differentiation in regenerative medicine.

\section{Nanomaterials as 3D nano-scaffolds for stem cell differentiation}

Although classical 2D cell cultures on flat surfaces of nanomaterials can manipulate the fates of stem cells, cells proliferation and differentiation inside the body are within complex 3D microenvironments. More and more current research on the differentiation of stem cells by nanomaterials are mainly focus on 3D environments, the nanomaterials-based 3D nano-scaffolds can simulate the natural environment and usually serve the purposes of assisting cell growth, cell attachment and specific differentiation. Previous study has shown that the stiffness and chemical composition of the scaffolds mainly affect the proliferation and differentiation capacity of stem cells [51]. Nanomaterials-based 3D scaffolds with different stiffness and chemical composition provide an ideal platform for cell-cell/nanomaterials communications and the properties of scaffolds can be varied to promote differentiation of stem cells into specific lineages [16].
3D nano-scaffold systems have proven to enhance osteogenic, neural, chondrogenic and odontogenic differentiation [89, 132-134].

\section{Application of nanomaterials in the differentiation of stem cells into specific lineages}

The application of various nanomaterials in stem cell differentiation has been mentioned above, and a summary is listed in Table 1. Combination of nanomaterials and stem cells brings us powerful tools to generate various specific lineages like osteoblast, neural cell, cardiocytes, chondrocyte, hepatocyte-like cells, and so on. It is noteworthy each nanomaterial is so versatile that can be fabricated for many purposes to differentiate different stem cells (MSCs, ESCs, ADSCs, NSCs, iPSCs, ect.) into different lineages. For example, silica nanoparticles have no positive effect on the differentiation of stem cells, but they can be designed as nanocarrier to carry insulin to rMSCs for adipogenic differentiation [94], or delivery AA to hESCs for cardiac differentiation [15]. Similarly, aligned SWCNTs have been reported to promote osteogenic differentiation of hMSCs [131]. In addition, carboxylated MWCNTs can direct neural differentiation of hBMSCs [86], and poly( $\varepsilon$-caprolactone)-functioned SWCNT scaffolds can enhance cardiac differentiation of rMSCs [90]. As shown in Table 1, AuNPs, AgNPs, silica nanoparticles and polymeric nanoparticles are more suitable as additives or as carriers for stem cell differentiation. However, $\mathrm{TiO}_{2}$, GR, GO and CNTs as 2D/3D nano-scaffolds for stem cell differentiation are more worthy of study and exploration.

Anyhow, in view of the inherent properties, ligands and the drug-loaded on the surface of nanomaterials have great influence on the differentiation, more research and efforts are needed to design suitable conditions for stem cell specific differentiation according to the characteristics of various nanomaterials.

\section{Conclusions}

The rapid development of nanotechnology provides a variety of nanomaterials, especially metal nanoparticles, carbon nanomaterials, semiconductor nanomaterials, polymeric nanoparticles and DNA nanostructures, which are promising in regulating stem cell behavior and tissue regeneration $[135,136]$. Nanomaterials can potently modulate the drug-loaded release or microenvironments involved in stem cell differentiation, and enhance their efficiency and safety [123]. The combination of stem cell research and nanomaterials offers new insights to treat various diseases, including cardiovascular disease, neurodegenerative diseases, bone tissue formation and regeneration $[33,129,137,138]$.

Among these nanomaterials, AuNPs are good conductors that can be used for deliver electrical stimulation on 
Table 1 A summary of the applications of nanomaterials in stem cell differentiation

\begin{tabular}{|c|c|c|c|c|}
\hline Nanomaterials & $\begin{array}{l}\text { Chemical modifications/ } \\
\text { components }\end{array}$ & Cell lineages generated & Cell sources & References \\
\hline \multicolumn{5}{|l|}{ Nanoparticles and nano-carriers } \\
\hline AuNPs & & Osteogenic differentiation & mMSCs/hADSCs/hMSCs & {$[34,44,47]$} \\
\hline AuNPs & Chitosan & Osteogenic differentiation & hADSCs & [49] \\
\hline AgNPs & & Osteogenic differentiation & mMSCs & [29] \\
\hline AgNPs & & Osteogenic differentiation & hUSCs & {$[57]$} \\
\hline AgNPs & miR-148b & Osteogenic differentiation & hADSCs & {$[58]$} \\
\hline GO & & Dopamine neurons & mESCs & {$[33]$} \\
\hline GQD & & Osteoblasts and adipocytes & rBMSCs & {$[79]$} \\
\hline Silica nanoparticles & Insulin & Adipogenic differentiation & rMSCs & {$[94]$} \\
\hline Silica nanoparticles & AA & Cardiac differentiation & hESCs & {$[15]$} \\
\hline Mesoporous silica nanoparticles & HNF3 $\beta$ plasmid DNA & Hepatocyte-like cells & miPSCs & [95] \\
\hline Silica nanoparticles & PEDF siRNA & Self-renewal and differentiation & hCSCs & {$[96]$} \\
\hline IONPS & & Osteogenic differentiation & hBMSCs & {$[65]$} \\
\hline IONPS & HSA/FGF2 & $\begin{array}{l}\text { Neuronal, adipogenic and } \\
\text { osteogenic lineages }\end{array}$ & hMSCs & {$[67]$} \\
\hline Barium titanate nanoparticles & & Proliferation and differentiation & rMSCs & {$[68]$} \\
\hline DNA nanotubes & Peptide RGDS & Neurons & mNSCs & [114] \\
\hline $\begin{array}{l}\text { Chitosan-based-microRNA nano- } \\
\text { particles }\end{array}$ & AntimiR-138 & Osteogenic differentiation & rMSCs & [104] \\
\hline Polymeric nanoparticles & RA & Neuronal differentiation & $\begin{array}{l}\text { mNSCs/hiPSCs/mouse SVZ stem } \\
\text { cells }\end{array}$ & {$[99,100,130]$} \\
\hline Chitosan nanoparticles & Hepatocyte growth factor & Hepatocytes & mBMSCs & [129] \\
\hline $\begin{array}{l}\text { Polyethyleneimine complex nano- } \\
\text { particles }\end{array}$ & RA & Neuronal differentiation & mESCs & [98] \\
\hline Polymeric nanoparticles & siSOX9 and RA & Neurons & mNSCs & [140] \\
\hline \multicolumn{5}{|l|}{ 2D and 3D nano-scaffolds } \\
\hline $\begin{array}{l}\text { AuNPs-loaded functionalized } \\
\text { nanofibers }\end{array}$ & $\begin{array}{l}\text { PCL/SF/AV/VitB12/GNP } \\
\text { fibers }\end{array}$ & Cardiac differentiation & hMSCs & {$[51]$} \\
\hline AuNPs-loaded hybrid nanofibers & BSA/PVA scaffolds & Cardiac differentiation & hMSCs & [46] \\
\hline $\mathrm{TiO}_{2}$ nanotubes & & Osteogenic differentiation & hADSCs/rBMSCs & {$[35,62]$} \\
\hline $\mathrm{TiO}_{2}$-coated CoCrMo & & Osteogenic differentiation & hMSCs & {$[141]$} \\
\hline $\mathrm{TiO}_{2}$ scaffolds & & Osteogenic differentiation & hADSCs & {$[14]$} \\
\hline $\mathrm{GR} / \mathrm{TiO}_{2}$ heterojunction & & Neurons & hNSCs & {$[142]$} \\
\hline GR & Laminin-coated & Neurons & hNSCs & {$[76]$} \\
\hline$G R$ & & Osteogenic differentiation & hMSCs & {$[41]$} \\
\hline GO-PLGA nanofiber scaffolds & & Osteogenic differentiation & hMSCs & {$[78]$} \\
\hline rGO-collagen hybrid scaffold & & Neural cells & rBMSCs & {$[77]$} \\
\hline Graphene nanogrids & & Osteogenic differentiation & hMSCs & [143] \\
\hline Aligned SWCNTS & & Osteogenic differentiation & hMSCs & [131] \\
\hline SWCNTS & & Adipogenesis & rMSCs & {$[87]$} \\
\hline MWCNTs & Poly(ع-caprolactone) & Cardiac differentiation & hMSCs & {$[90]$} \\
\hline MWCNTS & Carboxylated & Neural cells & hBMSCs & {$[86]$} \\
\hline MWCNTS & PEG & Osteogenic differentiation & hMSCs & {$[40]$} \\
\hline $\begin{array}{l}\text { MWCNTs-incorporated nanocom- } \\
\text { posite scaffolds }\end{array}$ & & Cartilage regeneration & hBMSCs & {$[88,89]$} \\
\hline $\begin{array}{l}\text { Xanthan and magnetite nanoparti- } \\
\text { cles hybrid scaffolds }\end{array}$ & & Neurons & mESCs & [144] \\
\hline PLLA/PBLG/collagen nanofibrous & & Osteogenic lineages & Rabbits-ADSCs & [145] \\
\hline
\end{tabular}


the differentiation of stem cells. Some other nanoparticles, such as AgNPs, silica nanoparticles, PEI, chitosan and DNA nanostructures, seem to have more advantages in drug delivery. However, due to most of the nanomaterials mentioned above are not biodegradable, chronic toxicity and other side effects should be noted when nanoparticles are used as supplements or nanocarriers for stem cell differentiation. Therefore, two kinds of biodegradable nanomaterials, chitosan and DNA nanostructures, are worth exploring as a nanocarrier for drug delivery. Moreover, $\mathrm{TiO}_{2}, \mathrm{GO}, \mathrm{GR}$ and CNTs are ideal biocompatible and mechanically platforms that are worth exploring for 2D matrix supports or 3D nano-scaffolds to facilitate stem cell differentiation. Moreover, some nanomaterials exhibit concentration-dependent toxicity, which should be taken into account during the application of nanomaterials $[52,139]$.

As mentioned above, the application of nanomaterials in the modulation of stem cell differentiation mainly through three ways (nanoparticle suspension, 2D culture, 3D culture), and the functions and mechanisms of nanomaterials in the differentiation of stem cells are different. In addition to the inherent ability to promote stem cell differentiation, nanomaterials with special desired lineages or drug loadings will modulate the specific properties for stem cell differentiation, and its stiffness, alignment and several other parameters also proved to play an important role in stem cell fate. Because of its complexity, the exact mechanisms linking the nanomaterials and the fate of stem cells are not well studied. Most of the literatures have not been deeply studied on the mechanism of the differentiation of stem cells promoted by nanomaterials. Further researches are hence needed to elucidate the mechanisms and biological effects of nanomaterials on stem cell differentiation. In addition, in order to improve the cell response for specific differentiation, novel nanomaterials with appropriate nanobio-interface, specific physical, biochemical, and biomechanical cues also should be further developed.

Based on the main findings from above mentioned studies, it is reasonable to believe that the differentiation of stem cells modulated by nanomaterials has broad application prospects. Although the combined use of stem cells and nanomaterials in stem cell differentiation currently is still in preliminary research phase and far from being applied clinically, and there are still many challenges to be solved in the use of nanomaterials for stem cell differentiation, this strategy is still promising for the application of stem cell differentiation in stem cell therapy and will certainly have a breakthrough in the recent future.

\section{Abbreviations}

SSCs: somatic stem cells; iPSCs: induced pluripotent stem cells; MSCs: mesenchymal stem cells; ADSCs: adipose-derived stem cells; NSCs: neural stem cells; BMSCs: bone-derived mesenchymal stem cells; USCs: urine-derived stem cells; $\mathrm{TiO}_{2}$ : titanium dioxide nanoparticles; AuNPs: gold nanoparticles; AgNPs: silver nanoparticles; GR: graphene; GO: graphene oxide; IONPs: iron oxide nanoparticles; GQD: graphene quantum dots; CNTs: carbon nanotubes; MAPK: mitogen-activated protein kinase; HSCs: hematopoietic stem cells; BMP2: bone morphogenetic protein 2; RGDS: Arg-Gly-Asp-Ser; 2D: two-dimensional; 3D: three-dimension; HAS: human serum albumin; FGF2: fibroblast growth factor 2; PLGA: poly(lactic-co-glycolic acid); PEG: polyethylene glycol; HNF3ß: hepatocyte nuclear factor 3B; PEDF: epithelium-derived factor; RA: retinoic acid; SVZ: subventricular zone; HIF-1a: hypoxia inducible factor-1a; AA: ascorbic acid.

\section{Authors' contributions}

All authors participated in the planning, design and writing of the manuscript. All authors read and approved the final manuscript.

\section{Author details}

${ }^{1}$ Liaoning Provincial Center for Clinical Research on Neurological Diseases, The First Affiliated Hospital, Dalian Medical University, Dalian 116021, People's Republic of China. ${ }^{2}$ Liaoning Provincial Key Laboratory for Research on the Pathogenic Mechanisms of Neurological Diseases, The First Affiliated Hospital, Dalian Medical University, Dalian 116021, People's Republic of China. ${ }^{3}$ Collaborative Innovation Center for Brain Science, The First Affiliated Hospital, Dalian Medical University, Dalian 116021, People's Republic of China.

\section{Competing interests}

The authors declare that they have no competing interests.

\section{Ethical approval and consent to participate}

Ethical approval and consent to participate is not applicable to this article as no data were generated or analysed during the current study.

\section{Funding}

This work was supported by Grants from the National Natural Science Foundation of China (31400853, 81430021 and 81370470), the Program for Liaoning Innovative Research Team in University (LT2015009).

\section{Publisher's Note}

Springer Nature remains neutral with regard to jurisdictional claims in published maps and institutional affiliations.

Received: 17 April 2017 Accepted: 14 October 2017

Published online: 25 October 2017

\section{References}

1. Meng X, et al. Endometrial regenerative cells: a novel stem cell population. J Transl Med. 2007;5:57.

2. Evans MJ, Kaufman MH. Establishment in culture of pluripotential cells from mouse embryos. Nature. 1981;292:154-6.

3. Thomson JA, et al. Embryonic stem cell lines derived from human blastocysts. Science. 1998;282:1145-7.

4. Takahashi K, Yamanaka S. Induction of pluripotent stem cells from mouse embryonic and adult fibroblast cultures by defined factors. Cell. 2006;126:663-76

5. Okita K, Ichisaka T, Yamanaka S. Generation of germline-competent induced pluripotent stem cells. Nature. 2007:448:313-7.

6. Yu J, et al. Induced pluripotent stem cell lines derived from human somatic cells. Science. 2007;318:1917-20.

7. Mcmurray RJ, Dalby MJ, Tsimbouri PM. Using biomaterials to study stem cell mechanotransduction, growth and differentiation. J Tissue Eng Regen Med. 2015;9:528-39.

8. Witkowska-Zimny M, et al. Effect of substrate stiffness on differentiation of umbilical cord stem cells. Acta Biochim Pol. 2012;59:261-4. 
9. Wu CH, et al. The isolation and differentiation of human adiposederived stem cells using membrane filtration. Biomaterials. 2012;33:8228-39.

10. Caiazzo M, et al. Direct generation of functional dopaminergic neurons from mouse and human fibroblasts. Nature. 2011:476:224-7.

11. Lee MH, et al. ECM microenvironment unlocks brown adipogenic potential of adult human bone marrow-derived MSCs. Sci Rep. 2016;6:21173.

12. Lo B, Parham L. Ethical issues in stem cell research. Endocr Rev. 2009;30:204-13.

13. Cha C, Liechty WB, Khademhosseini A, Peppas NA. Designing biomaterials to direct stem cell fate. ACS Nano. 2012;6:9353-8.

14. Pullisaar $\mathrm{H}$, et al. Alginate hydrogel enriched with enamel matrix derivative to target osteogenic cell differentiation in $\mathrm{TiO} 2$ scaffolds. J Tissue Eng. 2015;6:2041731415575870.

15. Ren M, Han Z, Li J, Feng G, Ouyang S. Ascorbic acid delivered by mesoporous silica nanoparticles induces the differentiation of human embryonic stem cells into cardiomyocytes. Mater Sci Eng C Mater Biol Appl. 2015;56:348-55.

16. Lee WC, et al. Origin of enhanced stem cell growth and differentiation on graphene and graphene oxide. ACS Nano. 2011;5:7334-41.

17. Wang $Y$, et al. Mifepristone-inducible caspase-1 expression in mouse embryonic stem cells eliminates tumor formation but spares differentiated cells in vitro and in vivo. Stem Cells. 2012:30:169-79.

18. Przyborski SA. Differentiation of human embryonic stem cells after transplantation in immune-deficient mice. Stem Cells. 2005:23:1242-50.

19. De M, Ghosh PS, Rotello VM. Applications of nanoparticles in biology. Adv Mater. 2008;20:4225-41.

20. Rivera Gil P, et al. Development of an assay based on cell counting with quantum dot labels for comparing cell adhesion within cocultures. Nano Today. 2011;6:20-7.

21. Rivera Gil P, Huhn D, Del Mercato LL, Sasse D, Parak WJ. Nanopharmacy: inorganic nanoscale devices as vectors and active compounds. Pharmacol Res. 2010;62:115-25.

22. Cabrera I, et al. a-Galactosidase-A loaded-nanoliposomes with enhanced enzymatic activity and intracellular penetration. Adv Healthc Mater. 2016:5:829-40

23. Li X, et al. Autophagy-sensitized cytotoxicity of quantum dots in PC12 cells. Adv Healthc Mater. 2014;3:354-9.

24. Qiu J, et al. Fluorescent graphene quantum dots as traceable, $\mathrm{pH}$ sensitive drug delivery systems. Int J Nanomed. 2015;10:6709-24.

25. Ruenraroengsak $P$, et al. Translocation of functionalized multi-walled carbon nanotubes across human pulmonary alveolar epithelium: dominant role of epithelial type 1 cells. ACS Nano. 2016:10:5070-85.

26. Lv M, et al. Effect of graphene oxide on undifferentiated and retinoic acid-differentiated SH-SY5Y cells line. Nanoscale. 2012;4:3861-6.

27. Fenollosa R, et al. Silicon particles as trojan horses for potential cancer therapy. J Nanobiotechnol. 2014;12:35.

28. You DG, et al. ROS-generating $\mathrm{TiO} 2$ nanoparticles for non-invasive sonodynamic therapy of cancer. Sci Rep. 2016;6:23200.

29. Zhang $\mathrm{R}$, et al. Silver nanoparticles promote osteogenesis of mesenchymal stem cells and improve bone fracture healing in osteogenesis mechanism mouse model. Nanomedicine. 2015;11:1949-59.

30. Wei M, et al. Polyvalent immunostimulatory nanoagents with selfassembled CpG oligonucleotide-conjugated gold nanoparticles. Angew Chem Int Ed Engl. 2012;51:1202-6.

31. Simeonidis $\mathrm{K}$, et al. Regeneration of arsenic spent adsorbents by Fe/ MgO nanoparticles. J Chem Technol Biotechnol. 2017;92:1876-83.

32. Li J, Fan C, Pei H, Shi J, Huang Q. Smart drug delivery nanocarriers with self-assembled DNA nanostructures. Adv Mater. 2013;25:4386-96.

33. Yang $D$, et al. Graphene oxide promotes the differentiation of mouse embryonic stem cells to dopamine neurons. Nanomedicine (Lond). 2014;9:2445-55

34. Yi C, Liu D, Fong CC, Zhang J, Yang M. Gold nanoparticles promote osteogenic differentiation of mesenchymal stem cells through p38 MAPK pathway. ACS Nano. 2010;4:6439-48.

35. LV L, et al. The nanoscale geometry of $\mathrm{TiO} 2$ nanotubes influences the osteogenic differentiation of human adipose-derived stem cells by modulating H3K4 trimethylation. Biomaterials. 2015;39:193-205.
36. Pozio A, Palmieri A, Girardi A, Cura F, Carinci F. Titanium nanotubes stimulate osteoblast differentiation of stem cells from pulp and adipose tissue. Dent Res J (Isfahan). 2012;9:S169-74.

37. Tung JC, Paige SL, Ratner BD, Murry CE, Giachelli CM. Engineered biomaterials control differentiation and proliferation of human-embryonicstem-cell-derived cardiomyocytes via timed Notch activation. Stem Cell Rep. 2014;2:271-81.

38. Mashinchian $\mathrm{O}$, et al. Regulation of stem cell fate by nanomateria substrates. Nanomedicine (Lond). 2015:10:829-47.

39. Heng BC, Haider H, Sim EK, Cao T, Ng SC. Strategies for directing the differentiation of stem cells into the cardiomyogenic lineage in vitro. Cardiovasc Res. 2004;62:34-42.

40. Nayak TR, et al. Thin films of functionalized multiwalled carbon nanotubes as suitable scaffold materials for stem cells proliferation and bone formation. ACS Nano. 2010;4:7717-25.

41. Nayak TR, et al. Graphene for controlled and accelerated osteogenic differentiation of human mesenchymal stem cells. ACS Nano. 2011;5:4670-8.

42. Rosi NL, et al. Oligonucleotide-modified gold nanoparticles for intracellular gene regulation. Science. 2006;312:1027-30.

43. Giljohann DA, et al. Gold nanoparticles for biology and medicine. Angew Chem Int Ed Engl. 2010:49:3280-94.

44. Li J, et al. Gold nanoparticle size and shape influence on osteogenesis of mesenchymal stem cells. Nanoscale. 2016;8:7992-8007.

45. Carregal-Romero S, et al. NIR-light triggered delivery of macromolecules into the cytosol. J Control Release. 2012;159:120-7.

46. Ravichandran $\mathrm{R}$, et al. Gold nanoparticle loaded hybrid nanofibers for cardiogenic differentiation of stem cells for infarcted myocardium regeneration. Macromol Biosci. 2014;14:515-25.

47. Ko WK, et al. The effect of gold nanoparticle size on osteogenic differentiation of adipose-derived stem cells. J Colloid Interface Sci. 2015;438:68-76

48. Woo DG, et al. The effect of electrical stimulation on the differentiation of hESCs adhered onto fibronectin-coated gold nanoparticles. Biomaterials. 2009;30:5631-8.

49. Choi SY, et al. Gold nanoparticles promote osteogenic differentiation in human adipose-derived mesenchymal stem cells through the Wnt/ beta-catenin signaling pathway. Int J Nanomed. 2015;10:4383-92.

50. Li JJ, Kawazoe N, Chen G. Gold nanoparticles with different charge and moiety induce differential cell response on mesenchymal stem cell osteogenesis. Biomaterials. 2015;54:226-36.

51. Sridhar S, Venugopal JR, Sridhar R, Ramakrishna S. Cardiogenic differentiation of mesenchymal stem cells with gold nanoparticle loaded functionalized nanofibers. Colloids Surf B Biointerfaces. 2015:134:346-54.

52. Khanna P, Ong C, Bay BH, Baeg GH. Nanotoxicity: an interplay of oxidative stress, inflammation and cell death. Nanomaterials (Basel). 2015;5:1163-80

53. Lim PN, Chang L, Thian ES. Development of nanosized silver-substituted apatite for biomedical applications: a review. Nanomedicine. 2015;11:1331-44.

54. Samberg ME, Loboa EG, Oldenburg SJ, Monteiro-Riviere NA. Silver nanoparticles do not influence stem cell differentiation but cause minimal toxicity. Nanomedicine (Lond). 2012;7:1197-209.

55. Liu X, He W, Fang Z, Kienzle A, Feng Q. Influence of silver nanoparticles on osteogenic differentiation of human mesenchymal stem cells. J Biomed Nanotechnol. 2014;10:1277-85.

56. Sengstock C, Diendorf J, Epple M, Schildhauer TA, Koller M. Effect of silver nanoparticles on human mesenchymal stem cell differentiation. Beilstein J Nanotechnol. 2014;5:2058-69.

57. Qin $\mathrm{H}$, et al. Silver nanoparticles promote osteogenic differentiation of human urine-derived stem cells at noncytotoxic concentrations. Int J Nanomed. 2014;9:2469-78.

58. Qureshi AT, Monroe WT, Dasa V, Gimble JM, Hayes DJ. miR-148b-nanoparticle conjugates for light mediated osteogenesis of human adipose stromal/stem cells. Biomaterials. 2013;34:7799-810

59. Hou Y, et al. Effects of titanium nanoparticles on adhesion, migration, proliferation, and differentiation of mesenchymal stem cells. Int J Nanomed. 2013:8:3619-30.

60. Oh S, et al. Stem cell fate dictated solely by altered nanotube dimension. Proc Natl Acad Sci USA. 2009:106:2130-5. 
61. Park J, et al. TiO2 nanotube surfaces: $15 \mathrm{~nm}$ - an optimal length scale of surface topography for cell adhesion and differentiation. Small. 2009:5:666-71.

62. Yu W, Qian C, Jiang X, Zhang F, Weng W. Mechanisms of stem cell osteogenic differentiation on TiO nanotubes. Colloids Surf B Biointerfaces. 2015;136:779-85.

63. HuY, et al. TiO2 nanotubes as drug nanoreservoirs for the regulation of mobility and differentiation of mesenchymal stem cells. Acta Biomater. 2012:8:439-48.

64. Pullisaar $\mathrm{H}$, et al. Enhanced in vitro osteoblast differentiation on TiO2 scaffold coated with alginate hydrogel containing simvastatin. J Tissue Eng. 2013;4:2041731413515670.

65. Wang Q, et al. Response of MAPK pathway to iron oxide nanoparticles in vitro treatment promotes osteogenic differentiation of hBMSCs. Biomaterials. 2016:86:11-20.

66. Sun J, et al. Magnetic assembly-mediated enhancement of differentiation of mouse bone marrow cells cultured on magnetic colloidal assemblies. Sci Rep. 2014;4:5125.

67. Levy I, et al. Bioactive magnetic near Infra-Red fluorescent core-shell iron oxide/human serum albumin nanoparticles for controlled release of growth factors for augmentation of human mesenchymal stem cell growth and differentiation. J Nanobiotechnol. 2015;13:34

68. Ciofani $G$, et al. Effects of barium titanate nanoparticles on proliferation and differentiation of rat mesenchymal stem cells. Colloids Surf B Biointerfaces. 2013;102:312-20.

69. Shah B, Yin PT, Ghoshal S, Lee KB. Multimodal magnetic core-shell nanoparticles for effective stem-cell differentiation and imaging. Angew Chem Int Ed Engl. 2013;52:6190-5.

70. Wang Z, Dai Z. Carbon nanomaterial-based electrochemical biosensors: an overview. Nanoscale. 2015;7:6420-31.

71. Kong L, Chen W. Carbon nanotube and graphene-based bioinspired electrochemical actuators. Adv Mater. 2014;26:1025-43.

72. Zhou X, Liang F. Application of graphene/graphene oxide in biomedicine and biotechnology. Curr Med Chem. 2014;21:855-69.

73. Dreyer DR, Park S, Bielawski CW, Ruoff RS. The chemistry of graphene oxide. Chem Soc Rev. 2010;39:228-40.

74. Compton OC, Nguyen ST. Graphene oxide, highly reduced graphene oxide, and graphene: versatile building blocks for carbon-based materials. Small. 2010;6:711-23.

75. Chen GY, Pang DW, Hwang SM, Tuan HY, Hu YC. A graphene-based platform for induced pluripotent stem cells culture and differentiation. Biomaterials. 2012;33:418-27.

76. Park SY, et al. Enhanced differentiation of human neural stem cells into neurons on graphene. Adv Mater. 2011;23:H263-7.

77. Guo W, et al. Construction of a 3D rGO-collagen hybrid scaffold for enhancement of the neural differentiation of mesenchymal stem cells. Nanoscale. 2016;8:1897-904.

78. Luo Y, et al. Enhanced proliferation and osteogenic differentiation of mesenchymal stem cells on graphene oxide-incorporated electrospun poly(lactic-co-glycolic acid) nanofibrous mats. ACS Appl Mater Interfaces. 2015;7:6331-9.

79. Qiu J, et al. Effects of graphene quantum dots on the self-renewal and differentiation of mesenchymal stem cells. Adv Healthc Mater. 2016:5:702-10.

80. Quintana $M$, et al. A simple road for the transformation of few-layer graphene into MWNTs. J Am Chem Soc. 2012;134:13310-5.

81. Gong H, Peng R, Liu Z. Carbon nanotubes for biomedical imaging: the recent advances. Adv Drug Deliv Rev. 2013;65:1951-63.

82. Mooney E, Dockery P, Greiser U, Murphy M, Barron V. Carbon nanotubes and mesenchymal stem cells: biocompatibility, proliferation and differentiation. Nano Lett. 2008;8:2137-43.

83. Liu D, Yi C, Zhang D, Zhang J, Yang M. Inhibition of proliferation and differentiation of mesenchymal stem cells by carboxylated carbon nanotubes. ACS Nano. 2010;4:2185-95.

84. Ahmadi $\mathrm{H}$, et al. Acute toxicity of functionalized single wall carbon nanotubes: a biochemical, histopathologic and proteomics approach Chem Biol Interact. 2017;275:196-209.

85. Cheng J, Meziani MJ, Sun YP, Cheng SH. Poly(ethylene glycol)conjugated multi-walled carbon nanotubes as an efficient drug carrier for overcoming multidrug resistance. Toxicol Appl Pharmacol. 2011;250:184-93.
86. Chen YS, Hsiue GH. Directing neural differentiation of mesenchymal stem cells by carboxylated multiwalled carbon nanotubes. Biomaterials. 2013:34:4936-44.

87. Lee $\mathrm{JH}$, et al. Random networks of single-walled carbon nanotubes promote mesenchymal stem cell's proliferation and differentiation. ACS Appl Mater Interfaces. 2015;7:1560-7.

88. Holmes B, Fang X, Zarate A, Keidar M, Zhang LG. Enhanced human bone marrow mesenchymal stem cell chondrogenic differentiation in electrospun constructs with carbon nanomaterials. Carbon. 2016;97:1-13

89. Holmes B, Castro NJ, Li J, Keidar M, Zhang LG. Enhanced human bone marrow mesenchymal stem cell functions in novel 3D cartilage scaffolds with hydrogen treated multi-walled carbon nanotubes. Nanotechnology. 2013;24:365102.

90. Crowder SW, et al. Poly(epsilon-caprolactone)-carbon nanotube composite scaffolds for enhanced cardiac differentiation of human mesenchymal stem cells. Nanomedicine (Lond). 2013;8:1763-76.

91. Liu Y, Zhao Y, Sun B, Chen C. Understanding the toxicity of carbon nanotubes. Acc Chem Res. 2013;46:702-13.

92. Maggini $L$, et al. Breakable mesoporous silica nanoparticles for targeted drug delivery. Nanoscale. 2016:8:7240-7.

93. Park MV, et al. In vitro developmental toxicity test detects inhibition of stem cell differentiation by silica nanoparticles. Toxicol Appl Pharmacol. 2009;240:108-16.

94. Liu D, et al. Biocompatible silica nanoparticles-insulin conjugates for mesenchymal stem cell adipogenic differentiation. Bioconjug Chem. 2010;21:1673-84

95. Chen W, Tsai PH, Hung Y, Chiou SH, Mou CY. Nonviral cell labeling and differentiation agent for induced pluripotent stem cells based on mesoporous silica nanoparticles. ACS Nano. 2013;7:8423-40.

96. Liang $\mathrm{H}$, et al. The roles of nanocarriers on pigment epithelium-derived factor in the differentiation of human cardiac stem cells. Cell Tissue Res. 2015;362:611-21.

97. LiY, Gao GH, Lee DS. Stimulus-sensitive polymeric nanoparticles and their applications as drug and gene carriers. Adv Healthc Mater. 2013;2:388-417.

98. Ku B, Kim JE, Chung BH, Chung BG. Retinoic acid-polyethyleneimine complex nanoparticles for embryonic stem cell-derived neuronal differentiation. Langmuir. 2013;29:9857-62.

99. Seo Hl, et al. Thermo-responsive polymeric nanoparticles for enhancing neuronal differentiation of human induced pluripotent stem cells. Nanomedicine. 2015;11:1861-9.

100. Maia J, et al. Controlling the neuronal differentiation of stem cells by the intracellular delivery of retinoic acid-loaded nanoparticles. ACS Nano. 2011;5:97-106.

101. Santos T, et al. Polymeric Nanoparticles to control the differentiation of neural stem cells in the subventricular zone of the brain. ACS Nano. 2012;6:10463-74.

102. Ragelle $H$, Vandermeulen $G$, Preat V. Chitosan-based siRNA delivery systems. J Control Release. 2013;172:207-18.

103. Chen X, et al. Nanoparticle delivery of stable miR-199a-5p agomir improves the osteogenesis of human mesenchymal stem cells via the HIF1a pathway. Biomaterials. 2015;53:239-50.

104. Wu G, et al. Improving the osteogenesis of rat mesenchymal stem cells by chitosan-based-microRNA nanoparticles. Carbohydr Polym. 2016;138:49-58.

105. Ji J, et al. Sphere-shaped nano-hydroxyapatite/chitosan/gelatin 3D porous scaffolds increase proliferation and osteogenic differentiation of human induced pluripotent stem cells from gingival fibroblasts. Biomed Mater. 2015:10:045005.

106. Merdan T, Kopecek J, Kissel T. Prospects for cationic polymers in gene and oligonucleotide therapy against cancer. Adv Drug Deliv Rev. 2002;54:715-58.

107. Ben David-Naim M, et al. Polymeric nanoparticles of siRNA prepared by a double-emulsion solvent-diffusion technique: physicochemical properties, toxicity, biodistribution and efficacy in a mammary carcinoma mice model. Biomaterials. 2017;145:154-67.

108. Fischer D, Li Y, Ahlemeyer B, Krieglstein J, Kissel T. In vitro cytotoxicity testing of polycations: influence of polymer structure on cell viability and hemolysis. Biomaterials. 2003;24:1121-31. 
109. Kean T, Roth S, Thanou M. Trimethylated chitosans as non-viral gene delivery vectors: cytotoxicity and transfection efficiency. J Control Release. 2005;103:643-53.

110. Kumar A, Vimal A. Why chitosan? From properties to perspective of mucosal drug delivery. Int J Biol Macromol. 2016;91:615-22.

111. Li J, et al. Self-assembled multivalent DNA nanostructures for noninvasive intracellular delivery of immunostimulatory $\mathrm{CpG}$ oligonucleotides. ACS Nano. 2011;5:8783-9.

112. Yan J, et al. Growth and origami folding of DNA on nanoparticles for high-efficiency molecular transport in cellular imaging and drug delivery. Angew Chem Int Ed Engl. 2015:54:2431-5.

113. You Z, et al. Regulation of vascular smooth muscle cell autophagy by DNA nanotube-conjugated mTOR siRNA. Biomaterials. 2015;67:137-50.

114. Stephanopoulos N, et al. Bioactive DNA-peptide nanotubes enhance the differentiation of neural stem cells into neurons. Nano Lett. 2015:15:603-9.

115. Ha SW, Weitzmann MN, Beck GR Jr. Bioactive silica nanoparticles promote osteoblast differentiation through stimulation of autophagy and direct association with LC3 and p62. ACS Nano. 2014;8:5898-910.

116. Zhao F, Zhao Y, Liu Y, Chang X, Chen C. Cellular uptake, intracellular trafficking, and cytotoxicity of nanomaterials. Small. 2011;7:1322-37.

117. Ilie I, llie R, Mocan T, Bartos D, Mocan L. Influence of nanomaterials on stem cell differentiation: designing an appropriate nanobiointerface. Int J Nanomed. 2012;7:2211-25.

118. Chithrani BD, Ghazani AA, Chan WC. Determining the size and shape dependence of gold nanoparticle uptake into mammalian cells. Nano Lett. 2006;6:662-8.

119. Nel A, Xia T, Madler L, Li N. Toxic potential of materials at the nanolevel. Science. 2006:311:622-7.

120. Rivera-Gil P, et al. The challenge to relate the physicochemical properties of colloidal nanoparticles to their cytotoxicity. Acc Chem Res. 2013:46:743-9.

121. Seong JM, et al. Stem cells in bone tissue engineering. Biomed Mater. 2010;5:062001.

122. Zavan B, et al. Hyaluronan based porous nano-particles enriched with growth factors for the treatment of ulcers: a placebo-controlled study. J Mater Sci Mater Med. 2009;20:235-47.

123. Ferreira L. Nanoparticles as tools to study and control stem cells. J Cell Biochem. 2009;108:746-52.

124. Lundqvist $\mathrm{M}$, et al. Nanoparticle size and surface properties determine the protein corona with possible implications for biological impacts. Proc Natl Acad Sci USA. 2008:105:14265-70.

125. Agrawal S, Zhao Q. Antisense therapeutics. Curr Opin Chem Biol. 1998;2:519-28.

126. Szuts EZ, Harosi FI. Solubility of retinoids in water. Arch Biochem Biophys. 1991;287:297-304

127. Mintzer MA, Simanek EE. Nonviral vectors for gene delivery. Chem Rev 2009;109:259-302.

128. Cui ZK, et al. Delivery of siRNA via cationic Sterosomes to enhance osteogenic differentiation of mesenchymal stem cells. J Control Release. 2015:217:42-52.

129. Pulavendran S, Rose C, Mandal AB. Hepatocyte growth factor incorporated chitosan nanoparticles augment the differentiation of stem cell into hepatocytes for the recovery of liver cirrhosis in mice. J Nanobiotechnol. 2011:9:15.
130. Ferreira $R$, et al. Retinoic acid-loaded polymeric nanoparticles enhance vascular regulation of neural stem cell survival and differentiation after ischaemia. Nanoscale. 2016:8:8126-37.

131. Namgung S, Baik KY, Park J, Hong S. Controlling the growth and differentiation of human mesenchymal stem cells by the arrangement of individual carbon nanotubes. ACS Nano. 2011;5:7383-90.

132. Karadzic l, et al. Effects of novel hydroxyapatite-based 3D biomaterials on proliferation and osteoblastic differentiation of mesenchymal stem cells.J Biomed Mater Res A. 2015:103:350-7.

133. Ebrahimi-Barough S, Hoveizi E, Norouzi Javidan A, Ai J. Investigating the neuroglial differentiation effect of neuroblastoma conditioned medium in human endometrial stem cells cultured on 3D nanofibrous scaffold. $J$ Biomed Mater Res A. 2015;103:2621-7.

134. Qu T, Liu X. Nano-structured gelatin/bioactive glass hybrid scaffolds for the enhancement of odontogenic differentiation of human dental pulp stem cells. J Mater Chem B Mater Biol Med. 2013:1:4764-72.

135. Dayem AA, et al. The potential of nanoparticles in stem cell differentiation and further therapeutic applications. Biotechnol J. 2016;11:1550-60

136. Carradori D, Eyer J, Saulnier P, Preat V, Des Rieux A. The therapeutic contribution of nanomedicine to treat neurodegenerative diseases via neural stem cell differentiation. Biomaterials. 2017:123:77-91.

137. Kang PL, et al. Nano-sized collagen I molecules enhanced the differentiation of rat mesenchymal stem cells into cardiomyocytes. J Biomed Mater Res A. 2013;101:2808-16.

138. Zhao C, Tan A, Pastorin G, Ho HK. Nanomaterial scaffolds for stem cell proliferation and differentiation in tissue engineering. Biotechnol Adv. 2013;31:654-68.

139. Senut MC, et al. Size-dependent toxicity of gold nanoparticles on human embryonic stem cells and their neural derivatives. Small. 2016;12:631-46.

140. Zhang $\mathrm{R}$, et al. Traceable nanoparticle delivery of small interfering rna and retinoic acid with temporally release ability to control neural stem cell differentiation for alzheimer's disease therapy. Adv Mater. 2016;28:6345-52.

141. Logan N, et al. TiO2-coated CoCrMo: improving the osteogenic differentiation and adhesion of mesenchymal stem cells in vitro. J Biomed Mater Res A. 2015:103:1208-17.

142. Akhavan O, Ghaderi E. Flash photo stimulation of human neural stem cells on graphene/ $/ \mathrm{iO} 2$ heterojunction for differentiation into neurons. Nanoscale. 2013;5:10316-26.

143. Akhavan O, Ghaderi E, Shahsavar M. Graphene nanogrids for selective and fast osteogenic differentiation of human mesenchymal stem cells. Carbon. 2013:59:200-11.

144. Glaser T, Bueno VB, Cornejo DR, Petri DF, Ulrich H. Neuronal adhesion, proliferation and differentiation of embryonic stem cells on hybrid scaffolds made of xanthan and magnetite nanoparticles. Biomed Mater. 2015;10:045002.

145. Ravichandran R, Venugopal JR, Sundarrajan S, Mukherjee S, Ramakrishna S. Precipitation of nanohydroxyapatite on PLLA/PBLG/Collagen nanofibrous structures for the differentiation of adipose derived stem cells to osteogenic lineage. Biomaterials. 2012;33:846-55.

\section{Submit your next manuscript to BioMed Central and we will help you at every step:}

- We accept pre-submission inquiries

- Our selector tool helps you to find the most relevant journal

- We provide round the clock customer support

- Convenient online submission

- Thorough peer review

- Inclusion in PubMed and all major indexing services

- Maximum visibility for your research

Submit your manuscript at www.biomedcentral com/submit 